\title{
Producción de forraje y competencia interespecífica del cultivo asociado de avena (Avena sativa) con vicia (Vicia sativa) en condiciones de secano y gran altitud
}

\section{Forage production and interspecific competition of oats (Avena sativa) and common vetch (Vicia sativa) association under dry land and high-altitude conditions

\author{
Francisco Espinoza-Montes ${ }^{1,2,4}$, Wilfredo Nuñez-Rojas ${ }^{1}$, Iraida Ortiz-Guizado ${ }^{3}$, \\ David Choque-Quispe ${ }^{2}$
}

\section{RESUMEN}

Se experimentó el cultivo asociado de avena (Avena sativa) y vicia común (Vicia sativa) en condiciones de secano, a $4035 \mathrm{~m}$ sobre el nivel del mar, para conocer su comportamiento y efectos en el rendimiento, calidad de forraje y competencia interespecífica. En promedio, el rendimiento de forraje verde, materia seca y calidad de forraje fueron superiores al del monocultivo de avena $(\mathrm{p}<0.05)$. El porcentaje de proteína cruda se incrementó en la medida que creció la proporción de vicia común en la asociación, acompañado de una disminución del contenido de fibra. En cuanto a los índices de competencia, el cultivo asociado de avena con vicia favorece el rendimiento relativo total de forraje $\left(\operatorname{LER}_{\text {total }}>1\right)$. Ninguna de las especies manifestó comportamiento agresivo $(A=0)$. Se observó mayor capacidad competitiva de la vicia común $(\mathrm{CR}>1)$ comparado con la capacidad competitiva de la avena.

Palabras clave: cultivo asociado; rendimiento; calidad de forraje; competencia interespecífica

\section{AbSTRACT}

The oats (Avena sativa) and common vetch (Vicia sativa) cultivated in association was evaluated under dry land conditions at $4035 \mathrm{~m}$ above sea level to determine its performance and effects on yield, forage quality and interspecific competition. On avera-

${ }^{1}$ Escuela Profesional de Zootecnia, Universidad Nacional del Centro del Perú, Huancayo, Perú

${ }^{2}$ Escuela Profesional de Ingeniería Ambiental, Universidad Nacional José María Arguedas, Andahuaylas, Perú

${ }^{3}$ Escuela Profesional de Agropecuaria y Desarrollo Sostenible, Universidad Nacional de Educación Enrique Guzmán y Valle, Lima , Perú

${ }^{4}$ E-mail: franciscoespinozamontes@gmail.com

Recibido: 3 de abril de 2018

Aceptado para publicación: 14 de septiembre de 2018 
ge, the yield of green forage, dry matter and quality of forage were higher than that of oat monoculture $(p<0.05)$. The percentage of crude protein increased as the proportion of common vetch in the association increased, accompanied by a decrease in fibre content. Regarding the indices of competition, the associated crop of oats with common vetch favours the total relative yield of forage $\left(\operatorname{LER}_{\text {total }}>1\right)$. None of the species showed aggressive behaviour $(\mathrm{A}=0)$ and greater competitive ability of the common vetch $(\mathrm{CR}>1)$ was observed compared with the competitive capacity of oats.

Key words: associated crop; yield; forage quality; interspecific competition

\section{INTRODUCCIÓN}

Los cereales forrajeros son de gran importancia en la alimentación del ganado por su alta producción de materia seca y bajo costo; sin embargo, son pobres en contenido de proteína. Las leguminosas forrajeras se utilizan en la nutrición del ganado por su alto contenido de proteínas a bajo costo, pero tienen un bajo rendimiento de materia seca (Eskandari et al., 2009). La asociación de ambos forrajes puede aumentar su valor nutricional y la producción de biomasa (Aguilar et al., 2013).

La Avena sativa es una gramínea forrajera temporal para corte, adaptada a una gran diversidad de pisos altitudinales en el espacio agrícola andino, desde los 2500 a 4000 msnm, y a climas variados (Argote y Halanoca, 2007). La Vicia sativa es una de las leguminosas forrajeras anuales más importantes a nivel mundial, debido a sus múltiples usos y alta calidad nutricional (Kim et al., 2015). Las leguminosas tienen capacidad para desarrollar nódulos en sus raíces y fijar nitrógeno en simbiosis con rhizobios compatibles (Graham y Vance, 2003), que es utilizado por la planta huésped o por cultivos asociados (Graham y Vance, 2000), siendo la tasa de fijación de nitrógeno de 1 a $2 \mathrm{~kg}$ por hectárea por día (Giller, 2001).

El cultivo asociado de cereales y leguminosas es importante para el desarrollo de sistemas de producción de alimentos sostenibles, particularmente en sistemas de cultivo con insumos externos limitados (Adesogan et al., 2002). Tiene potencial de mejorar la fertilidad del suelo mediante la adición de nitrógeno producto de la fijación y extracción del componente leguminosa (Hauggaard-Nilsen et al., 2001), de hacer uso eficiente de los recursos ambientales (Knudsen et al., 2004), de reducir daños causados por plagas, enfermedades y malezas (Sekamatte et al., 2003; Banik et al., 2006), así como de mejorar la producción de forraje y su calidad a través de los efectos complementarios de dos o más cultivos establecidos simultáneamente en la misma área de cultivo (Ross et al., 2004; Bingol et al., 2007; Lithourgidis et al., 2007).

Las especies dentro de sistemas de cultivo asociado se desarrollan y compiten entre sí por recursos existentes en el medio, lo que resulta en competencia interespecífica entre especies. Las plantas también compiten por los componentes abióticos del agroecosistema para sobrevivir, siendo los más frecuentes el agua, nutrientes, luz, $\mathrm{O} 2$ y CO2 (Atis et al., 2012). Para reconocer la competencia entre especies se han desarrollado diversos modelos matemáticos. Estos modelos fueron resumidos por Weigelt y Jolliffe (2003) y entre ellos se seleccionaron la relación equivalente de la tierra (LER), agresividad (A) y relación de competencia (CR), que son algunos de los índices de competencia frecuentemente utilizados para comparar la competencia de las especies en sistemas de cultivo asociados (Dhima et al., 2007; Yilmaz et al., 2008; Erol 
et al., 2009, Wahla et al., 2009; Rahetlah et al., 2010).

Con el propósito de incrementar la productividad y calidad del forraje por unidad de área en un sistema de cultivo con bajos insumos externos, en terrenos de secano y con el agua disponible solo en época de lluvia, se plantearon los siguientes objetivos: (i) evaluar el rendimiento y calidad del forraje del cultivo asociado de avena con vicia común, según la proporción de semillas, en tres momentos de cosecha y (ii) determinar los efectos de la incorporación de la vicia común como componente del sistema de cultivo asociado con avena, a través de los índices de competencia.

\section{Materiales y Métodos}

\section{Lugar de Estudio}

El estudio se llevó a cabo en el fundo «Chulumana», ubicado en el distrito de San Pedro de Cajas, Tarma, Perú, entre las coordenadas $11^{\circ} 15^{\prime} 05^{\prime \prime} \mathrm{S}$ y $75^{\circ} 51^{\prime} 33^{\prime \prime} \mathrm{O}$, en el extremo sur de la meseta de Bombón, a 4035 $\mathrm{m}$ sobre el nivel del mar. El lugar tiene una breve temporada de lluvias de diciembre a marzo y una larga estación seca de abril a noviembre, con precipitación media anual de $772.3 \mathrm{~mm}$ y temperatura media anual de $7.6^{\circ} \mathrm{C}$.

\section{Muestra y Diseño Experimental}

Se realizó un experimento del cultivo asociado de avena (Avena sativa $\mathrm{L}$ ) con vicia (Vicia sativa $\mathrm{L}$ ) en campo de productor. Las proporciones de semilla utilizadas se calcularon sobre la base de las tasas de siembra únicas recomendadas de $100 \mathrm{~kg} / \mathrm{ha}$ de avena y $60 \mathrm{~kg}$ /ha de vicia (Argote y Halanoca, 2007; Rahetlah et al., 2010). La estructura experimental consistió en parcelas de monocultivo y parcelas con diferentes proporciones de semilla a la siembra de dos especies diferentes (Connolly et al., 2001). La proporción de semillas de avena y vicia al momento de la siembra fueron: 100:00, 67:33, 50:50, 33:67 y 00:100 para los tratamientos PS1, PS2, PS3, PS4 y PS5, respectivamente. Se preparó un inóculo de Rhizobium leguminosarium mezclando $500 \mathrm{~g}$ de este con una concentración ajustada a $10^{8} \mathrm{ufc} / \mathrm{ml}$ para los $60 \mathrm{~kg}$ de vicia, y se inoculó en los tratamientos con vicia. Las cosechas se realizaron a 120,150 y 180 días.

El experimento se organizó en un diseño de bloques completos al azar con tres repeticiones, distribuidos en 45 parcelas de $4 \times 5 \mathrm{~m}$ cada una, en un área total de $960 \mathrm{~m}^{2}$. El experimento se instaló en una parcela de rotación de papa, que al análisis de suelo, en el laboratorio de suelos de la Estación Experimental Agraria Santa Ana, Huancayo, reportó $4.54 \%$ de materia orgánica (alto), $18.6 \mathrm{ppm}$ de fósforo (alto), $152.76 \mathrm{ppm}$ de potasio (bajo), calificado como suelo franco con $46 \%$ de arena, $32 \%$ de limo y $22 \%$ de arcilla. La siembra se realizó el 14 de noviembre de 2015 y el registro de datos de la producción se realizaron a los 120, 150 y 180 días después de la siembra. Se utilizó el software SPSS v. 21 (IBM 2012), para el análisis de varianza (ANOVA). Las diferencias entre las medias se compararon mediante la prueba de Tukey $(\mathrm{p}<0.05)$ para subconjuntos homogéneos (Steel y Torrie, 1996).

\section{Estimación del Rendimiento}

El rendimiento de forraje verde (FV) se determinó cosechando los cultivos a mano a nivel del suelo y pesando el material fresco con una balanza electrónica de $2 \mathrm{~g}$ de aproximación. Las muestras de las parcelas con cultivo asociado se volvieron a pesar luego de separar la avena y la vicia. Para determinar la producción por hectárea se multiplicó por 10000 el peso de la muestra por metro cuadrado.

El rendimiento de materia seca (MS) se determinó multiplicando el rendimiento de FV por el contenido de MS de la muestra respectiva. El porcentaje de MS se calculó por método del horno microondas, en una prime- 
ra etapa con una potencia máxima $(900 \mathrm{~W})$ por $5 \mathrm{~min}$ y en etapas subsiguiente a mediana potencia $(400 \mathrm{~W})$ por 1 min por vez hasta que la variación de peso resultase menor de $0.2 \mathrm{~g}$ (Crespo et al., 2007).

\section{Contenido de Proteína y Fibra}

El análisis del contenido de proteína y fibra se realizó en el Laboratorio de Evaluación Nutricional de Alimentos de la Universidad Nacional Agraria La Molina. Se determinó el contenido de nitrógeno mediante el procedimiento micro-Kjeldahl (Nathan y Sun, 2006) y la concentración de proteína cruda (PC) se calculó con N x 6.25. El contenido de fibra neutra detergente (NDF) se estimó por el método Van Soest (Mertens, 2002).

\section{Competencia Interespecífica}

Para evaluar la competencia entre dos especies asociadas en un mismo campo de cultivo, se tomaron en cuenta los índices de competencia de plantas (Weigelt y Jolliffe, 2003). En este estudio, el comportamiento competitivo de la avena y la vicia se determinó en términos de relación equivalente de la tierra (LER), agresividad (A) y relación competitiva (CR).

\section{Relación Equivalente de la Tierra}

Con el fin de conocer el rendimiento relativo de las parcelas con monocultivo, en comparación con las parcelas con cultivos asociados, se utilizó el índice de relación equivalente de la tierra (Yilmaz et al., 2015). Los valores de LER se estimaron, para la avena, la vicia y sus mezclas, de acuerdo con la siguiente ecuación: $\mathrm{LER}=\mathrm{LER}_{\text {avena }}+\mathrm{LER}_{\text {vicia }}$; $\mathrm{LER}_{\text {avena }}=\mathrm{Y}_{\mathrm{ai}} / \mathrm{Y}_{\mathrm{a}} ; \mathrm{LER}_{\text {vicia }}=\mathrm{Y}_{\mathrm{vi}}^{\text {avena }} / \mathrm{Y}_{\mathrm{v}}$, donde $\mathrm{Y}_{\mathrm{a}}$ es el rendimiento de la avena como monocultivo, $\mathrm{Y}_{\mathrm{ai}}$ es el rendimiento de la avena como cultivo asociado, $Y_{v}$ es el rendimiento de la vicia como monocultivo e $\mathrm{Y}_{\mathrm{vi}}$ es el rendimiento de la vicia como cultivo asociado.
LER $>1$ indica que el cultivo asociado favorece el rendimiento de las especies. Por el contrario, $L E R<1$ indica que no hay ventaja del cultivo asociado y la competencia intraespecífica es más fuerte que la interacción interespecífica dentro del sistema de cultivos asociados (Dhima et al., 2007) y resulta en una mayor eficiencia en el uso del suelo (Zhang et al., 2011).

\section{Agresividad}

La agresividad (A) es un índice que se utiliza para indicar la dominancia relativa que tendría un cultivo «a» sobre un cultivo «b» en un sistema de cultivo asociado. Un valor de agresividad de cero indica que los cultivos asociados son igualmente competitivos; sin embargo, ambos cultivos podrían tener el mismo valor numérico, pero el signo de la especie dominante será positivo y del otro negativo; así mismo, cuanto mayor sea el valor numérico, mayores serán las diferencias entre los rendimientos reales (Wahla et al. 2009).

La agresividad se estimó de acuerdo con la siguiente ecuación: $A_{\text {avena }}=\left[\mathrm{Y}_{\mathrm{ai}} /\right.$ $\left.\left(\mathrm{Y}_{\mathrm{a}}^{*} \mathrm{P}_{\mathrm{ai}}\right)\right]-\left[\mathrm{Y}_{\mathrm{vi}} /\left(\mathrm{Y}_{\mathrm{v}} * \mathrm{P}_{\mathrm{vi}}\right)\right] ; \mathrm{A}_{\mathrm{vicia}}=\left[\mathrm{Y}_{\mathrm{vi}} /\right.$ $\left.\left(\mathrm{Y}_{\mathrm{v}} * \mathrm{P}_{\mathrm{vi}}\right)\right]-\left[\mathrm{Y}_{\mathrm{ai}} /\left(\mathrm{Y}_{\mathrm{a}} * \mathrm{P}_{\mathrm{ai}}\right)\right]$, donde $\mathrm{P}_{\mathrm{ai}}$ es la proporción sembrada de avena en asociación y $\mathrm{P}_{\mathrm{vi}}$ es la proporción sembrada de vicia en la asociación.

\section{Relación de Competencia}

La relación de competencia (CR) es un método utilizado para evaluar la competencia interespecífica entre los componentes de una asociación, dando una estimación de la capacidad competitiva de los cultivos asociados (Dhima et al., 2007). La CR se calculó de acuerdo con la siguiente ecuación: $\mathrm{CR}_{\text {avena }}$ $=\left[\mathrm{Y}_{\mathrm{ai}} /\left(\mathrm{Y}_{\mathrm{a}} * \mathrm{P}_{\mathrm{ai}}\right)\right] /\left[\mathrm{Y}_{\mathrm{vi}} /\left(\mathrm{Y}_{\mathrm{v}} * \mathrm{P}_{\mathrm{vi}}\right)\right] ; \mathrm{CR}_{\mathrm{vicia}} \stackrel{\text { avena }}{=}$ $\left[\mathrm{Y}_{\mathrm{vi}} /\left(\mathrm{Y}_{\mathrm{v}} * \mathrm{P}_{\mathrm{vi}}\right)\right] /\left[\mathrm{Y}_{\mathrm{ai}} /\left(\mathrm{Y}_{\mathrm{a}} * \mathrm{P}_{\mathrm{ai}}\right)\right]$. En un sistema de cultivo asociado, cuando $\mathrm{CR}_{\mathrm{xy}}>1$, la capacidad competitiva del cultivo «XX» es más alta que aquella del cultivo «y». 


\section{Resultados y Discusión}

\section{Rendimiento de Forraje Verde}

El cultivo asociado de avena con vicia favorece el rendimiento de forraje verde comparado con el rendimiento de forraje verde del monocultivo de avena, en las tres proporciones de semilla y en los tres momentos de cosecha.

De acuerdo con el Cuadro 1, el rendimiento de FV ha sido influenciado significativamente por la asociación del cereal con la leguminosa $(\mathrm{p}<0.05)$. El mayor rendimiento de FV fue de $16.58 \mathrm{t} /$ ha para PS3, superando al monocultivo de avena (PS1) en $13 \%$ y al monocultivo de vicia (PS5) en 36\%. Los rendimientos de FV de PS1, PS2 y PS4 fueron similares a la comparación estadística; sin embargo, se observa una diferencia numérica a favor de PS2 y PS4.

Se obtuvo un mayor rendimiento de FV cuanto mayor fue el intervalo entre la siembra y la cosecha $(p<0.05)$. La diferencia entre el rendimiento de 120 y 150 días fue de $1.63 \mathrm{t} / \mathrm{ha}$, con un incremento del rendimiento de $11.2 \%$, y la diferencia entre el rendimiento de 150 y 180 días fue de $0.98 \mathrm{t} / \mathrm{ha}$, con un incremento del rendimiento de $6.3 \%$. La opción de cosechar a una determinada edad dependerá del uso que se le dará al producto: forraje verde, heno o ensilado.

Los rendimientos de FV de este estudio fueron menores a los reportados por Lithourgidis et al. (2006) de 22.12 t/ha, Tuna y Orak (2007) de $25.3 \mathrm{t} / \mathrm{ha}$ y Ansar et al. (2010) de $37.97 \mathrm{t} / \mathrm{ha}$, para mezclas de 35:65, $75: 25$ y 50:50 de avena con vicia, respectivamente. Estos rendimientos difieren de los reportados para los monocultivos de avena y de vicia, donde Lithourgidis et al. (2006) señalan que el rendimiento del cultivo asociado es menor que el monocultivo de avena (33.23 $\mathrm{t} / \mathrm{ha}$ ) pero mayor al monocultivo de vicia (20.49 t/ha); de manera similar Ansar et al. (2010) refieren que el rendimiento del cultivo asociado es menor al del monocultivo de avena (31.58 t/ha) pero mayor al del monocultivo de vicia (13.76 t/ha); sin embargo, en el caso de Tuna y Orak (2007), el rendimiento del cultivo asociado fue mayor, tanto en el monocultivo de avena $(24.2 \mathrm{t} / \mathrm{ha})$ como en el monocultivo de vicia (19.6 t/ha). Los rendimientos de FV del monocultivo de avena varían entre 24.3 y 46.8 t/ha (Espitia et al., 2012) y el rendimiento de FV del monocultivo de vicia entre 5.0 t/ha (Aguilar et al., 2013) y 14.83 t/ha (Desalegn y Hassen, 2015).

\section{Rendimiento de Materia Seca}

El cultivo asociado de avena con vicia favorece el rendimiento de MS comparado con el rendimiento de MS del monocultivo de avena, en las tres proporciones de semilla y en los tres momentos de cosecha.

El rendimiento de MS que se presenta en el Cuadro 2, sigue una tendencia similar al rendimiento de FV. El rendimiento de MS ha sido influenciado significativamente por la asociación del cereal con la leguminosa $(\mathrm{p}<0.05)$. En promedio, el mayor rendimiento MS fue de $4.26 \mathrm{t} / \mathrm{ha}$ para PS3, superando al monocultivo de avena (PS1) en $6.3 \%$ y al monocultivo de vicia (PS5) en $44.6 \%$. Los rendimientos de MS de los tratamientos PS1 y PS2 y los tratamientos PS2 y PS4 fueron similares.

A mayor número de días de la siembra a la cosecha, mayor rendimiento de MS $(p<0.05)$. La diferencia entre el rendimiento de 120 y 150 días fue de $2.32 \mathrm{t} / \mathrm{ha}$, con un incremento del rendimiento de $58.6 \%$ y la diferencia entre el rendimiento de 150 y 180 días fue de $1.35 \mathrm{t} / \mathrm{ha}$, con un incremento del rendimiento de $25.4 \%$. La opción de cosechar a una determinada edad dependerá del uso que se le dará al producto: forraje verde, heno o ensilado.

Los rendimientos de MS de esta investigación son mayores a los reportados por Carr et al. (2004), quienes cultivando avena en tierras de secano y con bajos niveles de 
Cuadro 1. Efecto de la proporción de semilla y momento de cosecha sobre el rendimiento de forraje verde ( $t / h a)$ en el sistema de cultivo asociado de avena con vicia

\begin{tabular}{lcccc}
\hline \multirow{2}{*}{$\begin{array}{l}\text { Proporción de } \\
\text { semillas }\end{array}$} & \multicolumn{3}{c}{ Días entre la siembra y la cosecha } & \multirow{2}{*}{ Promedio } \\
\cline { 2 - 4 } & 120 & 150 & 180 & \\
\hline PS1 (100:00) & $13.50 \pm 0.90$ & $14.57 \pm 0.76$ & $15.17 \pm 0.68$ & $14.41^{\mathrm{b}}$ \\
PS2 (67:33) & $13.55 \pm 1.17$ & $15.52 \pm 0.51$ & $16.45 \pm 1.13$ & $15.17^{\mathrm{b}}$ \\
PS3 (50:50) & $14.82 \pm 0.56$ & $16.95 \pm 0.39$ & $17.98 \pm 0.21$ & $16.58^{\mathrm{a}}$ \\
PS4 (33:67) & $13.57 \pm 0.95$ & $14.98 \pm 0.91$ & $16.47 \pm 0.72$ & $15.01^{\mathrm{b}}$ \\
PS5 (00:100) & $9.32 \pm 0.55$ & $10.85 \pm 0.63$ & $11.75 \pm 0.78$ & $10.64^{\mathrm{c}}$ \\
\hline Promedio & $12.95^{\mathrm{c}}$ & $14.57^{\mathrm{b}}$ & $15.56^{\mathrm{a}}$ & \\
\hline
\end{tabular}

abc Letras diferentes en columnas y filas indican diferencias estadísticas $(p<0.05)$

Cuadro 2. Efecto de la proporción de semillas y momento de cosecha sobre el rendimiento de forraje en MS (t/ha), en el sistema de cultivo asociado de avena con vicia

\begin{tabular}{|c|c|c|c|c|}
\hline \multirow{2}{*}{$\begin{array}{l}\text { Proporción de } \\
\text { semillas }\end{array}$} & \multicolumn{3}{|c|}{ Días entre la siembra y la cosecha } & \multirow{2}{*}{ Promedio } \\
\hline & 120 & 150 & 180 & \\
\hline PS1 (100:00) & $2.07 \pm 0.13$ & $3.98 \pm 0.21$ & $5.95 \pm 0.27$ & $3.99^{\mathrm{ab}}$ \\
\hline PS2 (67:33) & $1.87 \pm 0.18$ & $4.42 \pm 0.17$ & $5.53 \pm 0.43$ & $3.94^{\mathrm{bc}}$ \\
\hline PS3 (50:50) & $1.82 \pm 0.07$ & $4.87 \pm 0.11$ & $6.08 \pm 0.07$ & $4.26^{\mathrm{a}}$ \\
\hline PS4 (33:67) & $1.55 \pm 0.11$ & $3.97 \pm 0.24$ & $5.32 \pm 0.29$ & $3.60^{\mathrm{c}}$ \\
\hline PS5 (00:100) & $0.89 \pm 0.05$ & $2.54 \pm 0.15$ & $3.66 \pm 0.25$ & $2.40^{\mathrm{d}}$ \\
\hline Promedio & $1.64^{\mathrm{c}}$ & $3.96^{\mathrm{b}}$ & $5.32^{\mathrm{a}}$ & \\
\hline
\end{tabular}

Cuadro 3. Efecto de la proporción de semillas y momento de cosecha sobre el contenido de proteína (PC) y fibra (NDF) en $\mathrm{g} / \mathrm{kg}$, en el sistema de cultivo asociado de avena con vicia

\begin{tabular}{ccccccc}
\hline Proporción de & \multicolumn{2}{c}{ 120 días } & \multicolumn{2}{c}{ 150 días } & \multicolumn{2}{c}{ 180 días } \\
\cline { 2 - 7 } semillas & PC & NDF & PC & NDF & PC & NDF \\
\hline PS1 (100:00) & 96.2 & 344.2 & 68.4 & 386.2 & 62.5 & 434.5 \\
PS2 (67:33) & 135.8 & 329.8 & 112.5 & 362.9 & 104.6 & 394.8 \\
PS3 (50:50) & 167.6 & 332.6 & 143.2 & 349.6 & 127.2 & 391.3 \\
PS4 (33:67) & 179.4 & 312.2 & 157.8 & 341.2 & 148.5 & 383.2 \\
PS5 (00:100) & 223.5 & 296.2 & 203.2 & 315.8 & 192.2 & 352.4 \\
\hline
\end{tabular}

Fuente: Laboratorio de Evaluación Nutricional de Alimentos, UNALM 
nitrógeno, obtienen $3.84 \mathrm{t} / \mathrm{ha}$ y asociando avena con guisante incrementan a $4.56 \mathrm{t} / \mathrm{ha}$. Así mismo, Desalegn y Hassen (2015) registran $2.79 \mathrm{t} / \mathrm{ha}$ en monocultivo de vicia.

Los rendimientos de MS obtenidos son menores a los reportados por Ansar et al. (2010) de 9.28 t/ha, Lithourgidis et al. (2006) de $9.22 \mathrm{t} / \mathrm{ha}$, Rahetlah et al. (2010) de $7.64 \mathrm{t} / \mathrm{ha}$, Tuna y Orak (2007) de $6.5 \mathrm{t} / \mathrm{ha}$ y Erol et al. (2009) de 6.32 t/ha, para mezclas de 65:35, 50:50, 35:65, 50:50, 75:25 y 45:55 de avena con vicia, respectivamente. Estos rendimientos difieren de los reportados para los monocultivos de avena y de vicia, donde Erol et al. (2009), Ansar et al. (2010) y Rahetlah et al. (2010) refieren que el rendimiento del cultivo asociado es mayor al del monocultivo de avena y al monocultivo de vicia, en tanto que Lithourgidis et al. (2006) y Tuna y Orak (2007) señalan que el rendimiento del cultivo asociado es menor que el monocultivo de avena, pero mayor al monocultivo de vicia. Para lograr mayores rendimientos de MS, los autores mencionados indican haber utilizado diferentes niveles de fertilización, especialmente fuentes de nitrógeno y fosforo.

\section{Calidad del Forraje}

El cultivo asociado de avena con vicia favorece la calidad del forraje comparado con la calidad del forraje del monocultivo de avena, en las tres proporciones de semilla y en los tres momentos de cosecha.

El resultado de la evaluación del valor nutricional se presenta en el Cuadro 3, donde se muestra que la proporción de semillas PS4, de la asociación de avena con vicia a 120 días tiene el más alto contenido de proteína $(179.4 \mathrm{~g} / \mathrm{kg})$ y el más bajo contenido de fibra $(312.2 \mathrm{~g} / \mathrm{kg})$. El contenido de proteína es mayor con relación al monocultivo de avena $(96.2 \mathrm{~g} / \mathrm{kg})$ y menor con relación al monocultivo de vicia $(223.5 \mathrm{~g} / \mathrm{kg})$. El contenido de fibra es mayor con relación al monocultivo de avena $(344.2 \mathrm{~g} / \mathrm{kg})$, y menor con relación al monocultivo de vicia $(296.2 \mathrm{~g} / \mathrm{kg})$. Se puede observar que los niveles de PC disminu- yen y los niveles de NDF incrementan en la medida que transcurre los días después de la siembra.

Estos resultados obtenidos en el estudio para PC y NDF son mayores que los reportados por Flores et al. (2016), quienes refieren que el patrón 65:35 de avena con vicia tiene el más alto contenido de $\mathrm{PC}(61 \mathrm{~g} / \mathrm{kg})$ y el patrón 35:65 el más alto contenido de NDF (407 g/kg); el contenido de PC es menor con relación al monocultivo de avena $(65 \mathrm{~g} / \mathrm{kg})$ y mucho menor con relación al monocultivo de vicia $(294 \mathrm{~g} / \mathrm{kg})$; el contenido de NDF es menor con relación al monocultivo de avena $(478 \mathrm{~g} / \mathrm{kg})$, pero mayor con relación al monocultivo de vicia $(363 \mathrm{~g} / \mathrm{kg})$. De igual forma Lithourgidis et al. (2006) señalan que el patrón 35:65 de avena con vicia, tiene el más alto contenido de PC $(119.1 \mathrm{~g} / \mathrm{kg})$ y el más alto contenido de NDF $(401.7 \mathrm{~g} / \mathrm{kg})$; el contenido de PC es mayor con relación al monocultivo de avena $(78.4 \mathrm{~g} / \mathrm{kg}$ ) y menor con relación al monocultivo de vicia $(139.3 \mathrm{~g} / \mathrm{kg})$; el contenido de NDF es mayor con relación al monocultivo de avena $(345.3 \mathrm{~g} / \mathrm{kg})$, y menor con relación al monocultivo de vicia (443.1 $\mathrm{g} / \mathrm{kg}$ ). Carr et al. (2004) refieren que asociando avena con guisante obtienen $100 \mathrm{~g} / \mathrm{kg}$ de PC con $552 \mathrm{~g} / \mathrm{kg}$ de NDF, superior al monocultivo de avena en el que obtienen $61 \mathrm{~g} / \mathrm{kg}$ de $\mathrm{PC}$ con $618 \mathrm{~g} / \mathrm{kg}$ de NDF. Así mismo, Desalegn y Hassen (2015) obtienen $91 \mathrm{~g} / \mathrm{kg}$ de PC con $441 \mathrm{~g} / \mathrm{kg}$ de NDF, y Aguilar et al. (2013) obtienen $234.2 \mathrm{~g} / \mathrm{kg}$ PC con 517.6 NDF en el cultivo de vicia en monocultivo.

\section{Relación Equivalente de la Tierra}

El cultivo asociado de avena con vicia favorece el rendimiento relativo total de forraje $\left(\operatorname{LER}_{\text {total }}>1\right)$, comparado con el rendimiento relativo por especie, en las tres proporciones de semilla y en los tres momentos de cosecha.

La LER parcial de la vicia aumentó en todos los casos a medida que disminuyó la proporción de avena en la mezcla (Cuadro 4). Las asociaciones tienen LER mayores a 
Cuadro 4. Efecto de la proporción de semillas y momento de cosecha sobre el rendimiento relativo total de forraje (LER) en el sistema de cultivo asociado de avena con vicia

\begin{tabular}{|c|c|c|c|c|c|c|c|c|c|}
\hline \multirow[b]{2}{*}{$\begin{array}{l}\text { Proporción } \\
\text { de semillas }\end{array}$} & \multicolumn{3}{|c|}{120 días } & \multicolumn{3}{|c|}{150 días } & \multicolumn{3}{|c|}{180 días } \\
\hline & 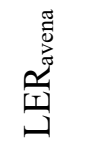 & 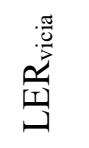 & 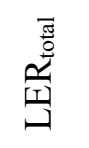 & 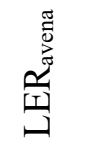 & 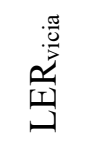 & 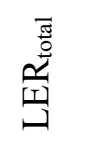 & 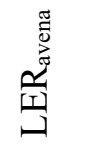 & 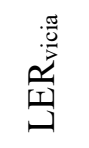 & 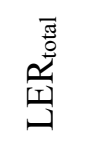 \\
\hline PS1 (100:00) & 1.00 & & 1.00 & 1.00 & & 1.00 & 1.00 & & 1.00 \\
\hline PS2 (67:33) & 0.75 & 0.36 & 1.12 & 0.76 & 0.42 & 1.17 & 0.75 & 0.43 & 1.18 \\
\hline PS3 (50:50) & 0.63 & 0.68 & 1.31 & 0.66 & 0.67 & 1.33 & 0.67 & 0.67 & 1.34 \\
\hline PS4 (33:67) & 0.38 & 0.90 & 1.28 & 0.42 & 0.81 & 1.24 & 0.43 & 0.85 & 1.28 \\
\hline PS5 (00:100) & & 1.00 & 1.00 & & 1.00 & 1.00 & & 1.00 & 1.00 \\
\hline
\end{tabular}

Cuando $L E R_{\text {total }}>1$ favorece el rendimiento relativo del cultivo asociado (Dhima et al., 2007)

Cuadro 5. Efecto de la proporción de semillas y momento de cosecha sobre la agresividad en el sistema de cultivo asociado de avena con vicia

\begin{tabular}{ccccccc}
\hline Proporción de & \multicolumn{2}{c}{ 120 días } & \multicolumn{2}{c}{150 días } & \multicolumn{2}{c}{180 días } \\
\cline { 2 - 7 } semillas & $\mathrm{A}_{\text {avena }}$ & $\mathrm{A}_{\text {vicia }}$ & $\mathrm{A}_{\text {avena }}$ & $\mathrm{A}_{\text {vicia }}$ & $\mathrm{A}_{\text {avena }}$ & $\mathrm{A}_{\text {vicia }}$ \\
\hline PS2 (67:33) & 0.000 & 0.000 & -0.001 & 0.001 & -0.002 & 0.002 \\
PS3 (50:50) & -0.001 & 0.001 & 0.000 & 0.000 & 0.001 & -0.001 \\
PS4 (33:67) & -0.002 & 0.002 & 0.000 & 0.000 & 0.000 & 0.000 \\
\hline
\end{tabular}

$A>0$ indica comportamiento agresivo; $A=0$ indica que no existe agresividad (Wahla et al., 2009)

Cuadro 6. Efecto de la proporción de semillas y momento de cosecha sobre la relación competitiva (CR) en el sistema de cultivo asociado de avena con vicia

\begin{tabular}{ccccccc}
\hline Proporción de & \multicolumn{2}{c}{120 días } & \multicolumn{2}{c}{150 días } & \multicolumn{2}{c}{180 días } \\
\cline { 2 - 7 } semillas & $\mathrm{CR}_{\text {avena }}$ & $\mathrm{CR}_{\text {vicia }}$ & $\mathrm{CR}_{\text {avena }}$ & $\mathrm{CR}_{\text {vicia }}$ & $\mathrm{CR}_{\text {avena }}$ & CR vicia \\
\hline PS2 (67:33) & 1.03 & 0.97 & 0.93 & 1.07 & 0.86 & 1.16 \\
PS3 (50:50) & 0.89 & 1.12 & 0.99 & 1.01 & 1.05 & 0.95 \\
PS4 (33:67) & 0.85 & 1.17 & 1.00 & 1.00 & 1.03 & 0.97 \\
\hline
\end{tabular}

CR>1: la capacidad competitiva de la especie es mayor con respecto a la otra (Zhang et al., 2011)

1.0, siendo mayores la LER del tratamiento PS3 a 120 días (1.31), 150 días (1.33) y 180 días de la siembra (1.34). El cultivo asociado de avena con vicia favorece el rendimiento del monocultivo de avena siendo el de mayor eficacia el patrón de cultivo PS3 (50:50).
Estos hallazgos concuerdan con los reportes de Dhima et al. (2007), quienes obtienen valores de LER relativamente menores (1.09) en la combinación 35:65 de avena con vicia. Erol et al. (2009) con bajos niveles de fertilización, reportan una mayor LER (1.33) 
con la mezcla 45:55 de avena con vicia, con una ventaja de $33 \%$ más de rendimiento, respecto al monocultivo de avena. Así mismo, Javanmard et al. (2014) indican que la mayor LER (1.16) se obtiene con la mezcla de 25:75 de avena con vicia, con una ventaja de $16 \%$ más de rendimiento, respecto al monocultivo de avena, mientras que Farías et al. (2016) indican que una mínima LER (1.18) se obtiene con la mezcla de $1: 1$ de avena con vicia, con una ventaja de $18 \%$ más de rendimiento, respecto al monocultivo de avena y recomienda una asociación prudente del 50\% de cada especie.

\section{Agresividad}

En el cultivo asociado de avena con vicia se observa que no se manifiesta comportamiento agresivo de ninguna de las especies $(\mathrm{A}=0)$ en las tres proporciones de semilla y en los tres momentos de cosecha. La tendencia de los valores obtenidos tiende a ser cero o cercanos a cero (Cuadro 5), lo que permite afirmar que no existe agresividad entre la avena y la vicia; por el contrario, la incorporación de la vicia como componente del cultivo asociado favorecería para lograr mayores rendimientos.

Contrariamente a estos resultados, Alemu et al. (2007) indican que la avena es agresiva con la vicia en cultivos asociados, y que la agresividad de la avena aumenta con el avance en la etapa de cosecha. Esto podría deberse a un crecimiento más rápido de la avena que le permite utilizar mejor los nutrientes y la energía solar en comparación con la vicia. Dhima et al. (2007) confirman esta versión al encontrar que la avena es la especie dominante, medido por el valor positivo de agresividad que presentan los patrones 45:55 (0.636) y 35:65 (0.337). Similares resultados son obtenidos por Rahetlah et al. (2010), medido por el valor positivo de agresividad que presenta el patrón 50:50 (0.71).

\section{Relación de Competencia de las Especies}

En el cultivo asociado de avena con vicia se observa mayor capacidad competitiva de la vicia $(\mathrm{CR}>1)$, comparado con la capacidad competitiva de la avena, dependiendo de las proporciones de semilla y los momentos de cosecha.

La capacidad competitiva de la vicia es variable según la proporción de semilla y el momento de cosecha (Cuadro 6). A los 120 días de la siembra es más competitiva en la proporción PS3 (50:50) y PS4 (33:67); a 150 días es más competitiva en las tres proporciones; y a 180 días solo es más competitiva en la proporción PS2 (67:33).

Los resultados concuerdan con los de Erol et al. (2009) quienes refieren que la CR de la vicia es mayor que la CR de la avena en las proporciones 75:25 (1.10) y 45:55 (1.17), y que la CR de la avena es mayor que la $\mathrm{CR}$ de la vicia en las proporciones 55:45 (1.24) y 25:75 (1.82). Para Dhima et al. (2007), los valores de CR para la avena fueron mayores que para la vicia, en las proporciones $45: 55$ (1.95) y 35:65 (1.38), lo que indica el predominio de las cereales en estas mezclas de cultivos; sin embargo, la CR de la avena disminuye a medida que la proporción de vicia aumenta, lo que indica que la avena es más competitiva que la vicia. También para Rahetlah et al. (2007), la CR para la avena fue mayor que para la vicia, en la proporción 50:50 (3.55), lo que indica el predominio del cereal en la mezcla con una leguminosa.

\section{Conclusiones}

- $\quad$ El rendimiento de forraje verde, materia seca y calidad del forraje fueron influenciados significativamente por la presencia de la leguminosa en el cultivo asociado de avena con vicia común, en condiciones de secano y gran altitud. 
- Los índices de competencia indican que la inclusión de la vicia común en el cultivo asociado favorece el rendimiento relativo total, que no se manifiesta comportamiento agresivo de ninguna de las especies y que la vicia común tendría mayor capacidad competitiva que la avena.

\section{Literatura Citada}

1. Adesogan AT, Salawu MB, Deaville E. 2002. The effect on voluntary feed intake, in vivo digestibility and nitrogen balance in sheep of feeding grass silage or pea-wheat intercrops differing in pea to wheat ratio and maturity. Anim Feed Sci Tech 96: 161-173. doi: 10.1016/ S0377-8401(01)00336-4

2. Aguilar EY, Bórquez JL, Domínguez IA, Morales A, Gutiérrez MG, González M. 2013. Forage yield, chemical composition and in vitro gas production of triticale (X Triticosecale wittmack) and barley (Hordeum vulgare) associated with common vetch (Vicia sativa) preserved as hay or silage. J Agr Sci 5: 227-238. doi: 10.5539/ jas.v5n2p227

3. Alemu B, Melaku S, Prasad NK. 2007. Effects of varying seed proportions and harvesting stages on biological compatibility and forage yield of oats (Avena sativa $\mathrm{L}$ ) and vetch (Vicia villosa $\mathrm{R}$ ) mixtures. Livestock Res Rural Develop 19(12). [Internet]. Available in: http://www.1rrd.org/lrrd19/1/ alem19012.htm

4. Ansar M, Ahmed ZI, Malik MA, Nadeem M, Majeed A, Rischkowsky $B A .2010$. Forage yield and quality potential of winter cereal-vetch mixtures under rainfed conditions. Emir J Food Agr 22:25-36. doi: 10.9755/ejfa.v22i1.-4904

5. Argote G, Halanoca M. 2007. Evaluación y selección de gramíneas forrajeras tolerantes a condiciones climáticas del altiplano de Puno. En: XX Reunión de la Asociación Latinoamericana de Producción Animal - ALPA. Cusco, Perú.
6. Atis I, Kokten K, Hatipoglu R, Yilmaz S, Atak M, Can E. 2012. Plant density and mixture ratio effects on the competition between common vetch and wheat. Aust J Crop Sci 6:498-505.

7. Banik P, Midya A, Sarkar BK, Ghose SS. 2006. Wheat and chickpea intercropping systems in an additive experiment. Advantages and weed smothering. Eur J Agron 24: 325-332. doi: 10.1016/j.eja.2005.10.010

8. Bingol N, Karsli T, Yilmaz IH, Bolat D. 2007. The effects of planting time and combination on the nutrient composition and digestible dry matter yield of four mixtures of vetch varieties intercropped with barley. Turk J Vet Anim Sci 31: 297-302.

9. Carr PM, Horsley RD, Poland WW. 2004. Barley, oat and cereal-pea mixtures as dryland forages in the Northern Great Plains. Agron J 96: 677-684.

10. Connolly J, Wayne P, Bazzaz FA. 2001. Interspecific competition in plants: how well do current methods answer fundamental questions? Am Nat 157: 107125. doi: $10.1086 / 318631$

11. Crespo R, Castaño J, Capurro J. 2007. Secado de forraje con el horno microondas: efecto sobre el análisis de calidad. Buenos Aires, Argentina. Agr Tec 67: 210-218. doi: 10.4067/S036528072007000200013

12. Desalegn K, Hassen W. 2015. Evaluation of biomass yield and nutritional value of different species of vetch (Vicia). Am Nat J Nutr 4: 99-105. doi: 10.5829/idosi.ajn.2015.4.3.96130

13. Dhima KV, Lithourgidis AS, Vasilakoglou IB, Dordas CA. 2007. Competition indices of common vetch and cereal intercrops in two seeding ratio. Field Crop Res 100: 249-256. doi: 10.1016/j.fcr.2006.07.008

14. Erol A, Kaplan M, Kizilsimsek M. 2009. Oats (Avena sativa) - common vetch (Vicia sativa) mixtures grown on a low-input basis for a sustainable agriculture. Trop Grasslands 43: 191-196 
15. Eskandari H, Ghanbari A, Javanmard A. 2009. Intercropping of cereals and legumes for forage production. Not Sci Biol 1: 7-13. doi: 10.15835/nsb113479

16. Espitia E, Villaseñor HE, Tovar R, Olán M, Limón A. 2012. Momento óptimo de corte para rendimiento y calidad de variedades de avena forrajera. Rev Mex Cienc Agr 3: 771-783.

17. Farías JA, Moraes PVD, Artuso VA, Dal JA, Da Silva DW, Kunz AG and Zanini $W$. 2016. Competitive effect between black oat (Avena strigosa) and common vetch (Vicia sativa) plants in consortium under diverse populational densities. J Agronomy 15: 184-190. doi: 10.3923/ja.2016.184.190

18. Flores MJ, Ricardo A. Sánchez RA, Echavarría FG, Gutiérrez, R, Rosales $C A$, Salinas $H$. 2016. Forage production and quality of common vetch mixtures with barley, oat and triticale in four phenological stages. Rev Mex Cienc Pecu 7: 275-291.

19. Giller KE. 2001. Nitrogen fixation in tropical cropping systems. Wallingford, UK: CABI Publishing. doi: 10.1079/ 9780851994178.0000

20. Graham PH, Vance CP. 2000. Nitrogen fixation in perspective: an overview of research and extension needs. Field Crops Res 65: 93-106. doi: 0.1104/pp.017004

21. Graham PH, Vance CP. 2003. Legumes: importance and constraints to greater use. Plant Physiol 131: 872-877. doi: 10.1104/pp.017004

22. Hauggaard-Nielsen H, Ambus P, Jensen ES. 2001. Interspecific competition, $\mathrm{N}$ use and interference with weeds in pea-barley intercropping. Field Crops Res 70: 101-109. doi: 10.1016/ S0378-4290(01)00126-5

23. IBM. 2012. SPSS Statistics 21 Brief Guide. USA: IBM Corporation. 170 p.

24. Javanmard A, Shekari F, Dehghanian H. 2014. Evaluation of forage yield and competition indices for intercropped barley and legumes. Int J Agric Biol Eng 8: 193-196.
25. Kim TS, Raveendar S, Suresh S, Lee GA, Lee JR, Cho JH, Lee SY, et al. 2015. Transcriptome analysis of two Vicia sativa subspecies: mining molecular markers to enhance genomic resources for vetch improvement. Genes 6: 1164-1182. doi: 10.3390/ genes6041164

26. Knudsen MT, Hauggaard-Nielsen H, Jornsgård B, Jensen ES. 2004. Comparison of interspecific competition and $\mathrm{N}$ use in pea-barley, faba beanbarley and lupin-barley intercrops grown at two temperate locations. Eur J Agron 142: 617-627. doi: 10.1017/S0021859604004745

27. Lithourgidis AS, Dhima KV, Vasilakoglou IB, Dordas CA Yiakoulaki MD. 2007. Sustainable production of barley and wheat by intercropping common vetch. Agron Sustain Dev 27: 95-99. doi: 10.1051/ agro:2006033

28. Lithourgidis AS, Vasilakoglou I, Dhima KV, Dordas CA, Yiakoulaki MD. 2006. Forage yield and quality of common vetch mixtures with oat and triticale in two seeding ratios. Field Crops Res 99: 106-113. doi: 10.1016/j.fcr.2006.03.008

29. Mertens DR. 2002. Gravimetric determination of amylase-treated neutral detergent fiber in feeds with refluxing in beakers or crucibles: collaborative study. J AOAC Int 85: 1217-1240.

30. Nathan M, Sun Y. 2006. Methods for plant analysis. A guide for conducting plant analysis in Missouri. USA: University of Missouri-Columbia. $19 \mathrm{p}$.

31. Rahetlah VB, Randrianaivoarivony JM, Razafimpamoa LH, Ramalanjaona $V L$. 2010. Effects of seeding rates on forage yield and quality of oat (Avena sativa $\mathrm{L}$ ) vetch (Vicia sativa $\mathrm{L}$ ) mixtures under irrigated conditions of Madagascar. Afr J Food Agric Nutr Dev 10:4254-4267.

32. Ross SM, King JR, Donovan JT, Spaner D. 2004. Intercropping berseem clover with barley and oat cultivars for 
forage. Agron J 96: 1719-1729. doi: 10.2134/agronj2004.1719

33. Sekamatte BM, Ogenga-Latigo M, Russell-Smith A. 2003. Effects of maize-legume intercrops on termite damage to maize, activity of predatory ants and maize yields in Uganda. Crop Prot 22: 87-93. doi: 10.1016/S02612194(02)00115-1

34. Steel RGD, Torrie JH. 1996. Bioestadística: principios y procedimientos. $2^{\mathrm{a}}$ ed. Mexico: McGraw-Hill. $640 \mathrm{p}$.

35. Tuna C, Orak A. 2007. The role of intercropping on yield potential of common vetch (Vicia sativa L) / oat (Avena sativa $\mathrm{L}$ ) cultivated in pure stand and mixtures. J Agric Biol Sci 2: 14-19.

36. Wahla IH, Ahmad R, Ehsanullah A, Jabbar A. 2009. Competitive functions of components crops in some barley based intercropping systems. Int J Agric
Biol 11: 69-72. doi: 11(1):1560-853011

37. Weigelt A, Jolliffe P. 2003. Indices of plant competition. J Ecology 91: 707-720. doi: 10.1046/j.1365-2745.2003.00805.x

38. YilmazS, Ali Özel A, Atak M, Erayman M. 2015. Effects of seeding rates on competition indices of barley and vetch intercropping systems in the Eastern Mediterranean. Turk J Agric For 39: 135 143. doi: 10.3906/tar-1406-155

39. Yilmaz S, Atak M, Erayman M. 2008. Identification of advantages of maizelegume intercropping over solitary cropping through competition indices in the East Mediterranean Region. Turk J Agric For 32: 111-119.

40. Zhang G, Yang Z, Dong S. 2011. Interspecific competitiveness affects the total biomass yield in an alfalfa and corn intercropping system. Field Crops Res 124: 66-73. doi: 10.1016/j.fcr.2011.06.006 